\title{
Hipocalemia refractaria como manifestación clínica de síndrome de Cushing ectópico: a propósito de dos casos y revisión de la literatura
}

\author{
Refractory Hypokalemia as a Clinical Manifestation of Ectopic Cushing \\ Syndrome: A Report of Two Cases and a Review of the Literature
}

Coronel Restrepo $N^{1}$, Mejía Zuluaga $M^{2}$, Aristizábal $N^{3}$, Torres Grajales $L^{4}$

${ }^{1}$ Médico Internista, Universidad CES; residente de Endocrinología, diabetes y metabolismo, Universidad Pontificia Bolivariana (UPB), Medellín, Colombia.

${ }^{2}$ Médico Internista, Universidad CES, Medellín, Colombia. ${ }^{3}$ Médica Internista y Endocrinóloga, Clínica Las Américas. Clínica Universitaria Bolivariana. Endocrino SAS. Docente del programa de Endocrinología, diabetes y metabolismo UPB. Medellín, Colombia.

${ }^{4}$ Médico Internista y Endocrinólogo, Clínica Las Américas. Endocrino SAS. Docente del programa de Endocrinología, diabetes $y$ metabolismo UPB. Medellín, Colombia.

Autor de contacto: Nicolás Coronel - Restrepo

Correo electrónico: ncoronel.restrepo@gmail.com

Fecha de recepción: 19/10/2018

Fecha de aceptación: 21/11/2018

\section{Resumen}

El síndrome de Cushing (SC) es un conjunto de signos y síntomas causado por el exceso de cortisol libre en el plasma. Sus manifestaciones clínicas son múltiples y en ocasiones poco específicas, haciendo difícil su sospecha clínica. La confirmación del hipercortisolismo es un proceso minucioso que debe hacerse ordenadamente para obtener un diagnóstico adecuado y preciso. A continuación se presentan dos casos de pacientes oncológicos cuya manifestación clínica paraneoplásica, por secreción ectópica de hormona adrenocorticotrópica (ACTH) y consecuente síndrome de Cushing asociado, fue una hipocalemia severa refractaria al tratamiento convencional. Siguiendo el abordaje presentado en las diferentes sociedades de endocrinología, se incluye un flujograma diagnóstico de pacientes con hipocalemia y una revisión actualizada de la literatura referente al SC ectópico.

Palabras clave: síndrome de Cushing, secreción ectópica de hormona adrenocorticotropa, hipocalemia, tumor neuroendocrino, alcalosis metabólica

\section{Abstract}

Cushing's syndrome (CS) is a set of signs and symptoms caused by the excess of free cortisol in the plasma. Its clinical manifestations are multiple and sometimes not very specific, making its clinical diagnosis difficult. The confirmation of hypercortisolism is a thorough out process that must be done in order to obtain an adequate and accurate diagnosis. The following are two cases of cancer patients whose paraneoplastic clinical manifestation, due to ectopic adrenocorticotropic hormone (ACTH) secretion and associated cushing syndrom, was a severe hypokalemia refractory to conventional treatment. Following the approach presented in the different endocrinology societies, a diagnostic flow chart of patients with hypokalemia is presented and an updated review of the literature regarding ectopic CS.

Key Words: Cushing syndrome, ectopic ACTH secretion, hypocalemia, neuro endocrine tumor, metabolic alcalosis.

\section{Introducción}

El diagnóstico de hipercortisolismo endógeno y sus consecuencias es un verdadero reto clínico a pesar de que llevamos más de 100 años desde la primera descripción que se hizo del síndrome de Cushing. No solo por el amplio espectro de signos y síntomas que conlleva la enfermedad, sino también por las diferentes formas clínicas que se presentan que hacen difícil un abordaje temprano (Ej. subclínico, cíclico, manifiesto). No existe un signo patognomónico de la enfermedad y la intensidad de los síntomas va a estar determinada por la magnitud y duración del hipercortisolismo, presencia o ausencia de androgenismo asociado, la etiología del mismo y la edad del paciente al momento de la presentación (síntomas menos severos en $>50$ años $)^{(1)}$. Desde la primera manifestación clínica (Ej. hipertensión arterial, obesidad, diabetes mellitus, osteoporosis, hipocalemia, alcalosis, entre otros) hasta la confirmación diagnóstica, pueden pasar alrededor de 3,8 años (promedio 
de 2,0 años $)^{(2)}$ y en el caso particular de tumores secretores de ACTH cerca del $20 \%$ de estos pueden permanecer ocultos durante muchos años más ${ }^{(3)}$. Su abordaje es siempre un reto diagnóstico para el médico, pues se debe clasificar para realizar un tratamiento adecuado, definitivo y oportuno ${ }^{(4)}$. A continuación, presentamos dos casos de síndrome de Cushing por secreción ectópica de ACTH como manifestación paraneoplásica atendidos en la Clínica Las Américas de la ciudad de Medellín, Colombia, en quienes la principal manifestación clínica fue una hipocalemia refractaria al tratamiento convencional. Posteriormente se hace una revisión actualizada del tema y se propone un flujograma diagnóstico de hipocalemia y sospecha de hipercortisolismo como fuente etiológica.

\section{Reporte de casos}

\section{Caso 1}

Hombre de 50 años con antecedente patológico de carcinoma de células pequeñas de pulmón metastásico a sistema nervioso central (SNC) en tratamiento paliativo, segunda línea de quimioterapia con topotecán y radioterapia holoencefálica. Sin evidencia de progresión tumoral en neuroimágenes de control. Como antecedente adicional refería haber presentado herpes zóster en dermatoma T4 izquierdo, tres meses atrás, el cual fue tratado satisfactoriamente con aciclovir. Secundario a las metástasis cerebrales tenía un síndrome convulsivo que era tratado con ácido valproico. Ingresa ahora al servicio de urgencias de Clínica Las Américas, Medellín, Colombia, por un cuadro clínico caracterizado por debilidad muscular en extremidades inferiores con progresión a miembros superiores y limitación funcional secundaria asociado a dificultad para la marcha pero sin compromiso respiratorio ni deglutorio. A la revisión por sistemas no refería fiebre, disnea, cefalea, alteraciones visuales, dolor lumbar o radicular. $\mathrm{Al}$ examen físico se encontraba con cifras elevadas de presión arterial sin criterios de urgencia hipertensiva, paresia de ambos miembros inferiores (fuerza muscular 4/5), parálisis facial izquierda, adiadococinesia y dismetría de ambos miembros superiores. Se realizó una resonancia magnética (RM) de cerebro contrastada, en la cual se evidenciaron múltiples nódulos intraxiales supra e infratentoriales en la secuencia de susceptibilidad, algunas presentaban componente hipointenso, sugiriendo áreas de sangrado o por calcificación por angeítis calcificante secundarias al antecedente de radioterapia. Algunas de las lesiones presentaban edema vasogénico mínimo y no había diferencia en progresión respecto a estudios previos. Se documentó adicionalmente hipocalemia asociada a alcalosis metabólica (tabla 1), por lo que se inició reposición de potasio por vía veno-

Tabla 1. Resumen de resultados de laboratorios casos clínicos 1 y 2

\begin{tabular}{|c|c|c|c|}
\hline \multicolumn{4}{|c|}{ Laboratorios iniciales } \\
\hline Bioquímica sanguínea & Caso 1 & Caso 2 & Valor de referencia \\
\hline Sodio & $144 \mathrm{mmol} / \mathrm{L}$ & $145 \mathrm{mmol} / \mathrm{L}$ & $(135-145)$ \\
\hline Magnesio & $1,96 \mathrm{mmol} / \mathrm{L}$ & $1,63 \mathrm{mmol} / \mathrm{L}$ & $(1,32-2,14)$ \\
\hline Calcio & $7,8 \mathrm{mg} / \mathrm{dl}$ & $7,5 \mathrm{mg} / \mathrm{dl}$ & $(8,8-10,4)$ \\
\hline Potasio & $2,1 \mathrm{mmol} / \mathrm{L}$ & $1,8 \mathrm{mmol} / \mathrm{lt}$ & $(3,5-5,5)$ \\
\hline Glucosa & $102 \mathrm{mg} / \mathrm{dl}$ & $156 \mathrm{mg} / \mathrm{dl}$ & $(70-100)$ \\
\hline Gases arteriales & $\begin{array}{c}\mathrm{pH}: 7,61 \\
\text { pCO2: } 39 \mathrm{mmHg} \\
\mathrm{HCO} 3: 59 \mathrm{mmol} / \mathrm{L} \\
\text { PO2: } 59 \mathrm{mmHg} \\
\text { FiO2: } 21 \%\end{array}$ & $\begin{array}{c}\mathrm{pH}: 7,61 \\
\mathrm{pCO} 234 \mathrm{mmHg} \\
\mathrm{HCO}: 33,5 \mathrm{mmol} / \mathrm{L} \\
\mathrm{PO} 2: 50 \mathrm{mmHg} \\
\text { FiO2: } 21 \%\end{array}$ & $\begin{array}{l}\mathrm{pH}: 7,36-7,44 \\
\text { pCO2: } 36-44 \\
\text { HCO3: } 22 \text { - } 26 \\
\text { PO2: } 85-100 \\
\text { FiO2: } 21 \%\end{array}$ \\
\hline Pruebas hormonales & Caso 1 & Caso 2 & Valor de referencia \\
\hline Cortisol 8 a.m. & $>60 \mathrm{mcg} / \mathrm{dl}$ & $57,79 \mathrm{mcg} / \mathrm{dl}$ & $(6-23)$ \\
\hline Corsisol en orina de $24 \mathrm{~h}$ & 253 mcg/día & 194,04 mcg /día & $(21-90)$ \\
\hline $\begin{array}{l}\text { Supresion de cortisol dosis bajas de } \\
\text { dexametasona ( } 1 \mathrm{mg} \text { a las } 23 \text { horas) }\end{array}$ & $>60 \mathrm{mcg} / \mathrm{dl}$ & $62 \mathrm{mcg} / \mathrm{dl}$ & $\begin{array}{l}\text { Síndrome de Cushing: Cortisol sérico } \\
\text { post dexametasona }>1,8 \mathrm{mcg} / \mathrm{dl}\end{array}$ \\
\hline ACTH & $765 \mathrm{pg} / \mathrm{ml}$ & $135,6 \mathrm{pg} / \mathrm{ml}$ & $(5-60)$ \\
\hline $\begin{array}{l}\text { Supresión de cortisol con dosis al- } \\
\text { tas de dexametasona ( } 8 \mathrm{mg} \text { a las } 23 \\
\text { horas) }\end{array}$ & $\begin{array}{c}\text { Basal: } \\
>60 \mathrm{mcg} / \mathrm{dl} \\
\text { Postsupresión: } \\
>60 \mathrm{mcg} / \mathrm{dl}\end{array}$ & $\begin{array}{c}\text { Basal: } \\
58,71 \mathrm{mcg} / \mathrm{dl} \\
\text { Postsupresión: } \\
63,7 \mathrm{mcg} / \mathrm{dl}\end{array}$ & $\begin{array}{l}\text { Enfermedad de Cushing: Cortisol sé- } \\
\text { rico postdexametasona }<5 \mathrm{mcg} / \mathrm{dl} \text { o } \\
\text { reducción }>50 \% \text { respecto a la basal }\end{array}$ \\
\hline
\end{tabular}


sa con $20 \mathrm{ml}$ de cloruro de potasio (dos ampollas) a través de catéter central de inserción periférica (PICC) cada seis horas, además gluconato de potasio $15 \mathrm{ml}$ vía oral cada seis horas y espironolactona $50 \mathrm{mg}$ vía oral cada día. Sin embargo, pasadas 48 horas de inicio del tratamiento, los niveles de potasio no aumentaban (potasio 2,2 meq/l), por lo que solicitan interconsulta al servicio de endocrinología. Dado el contexto clínico, se plantea la posibilidad diagnóstica de que pudiera tratarse de una manifestación paraneoplásica. La glucosa sérica fue de $102 \mathrm{mg} / \mathrm{dl}$ (60-100) y el cortisol 8 a.m. $>60 \mathrm{mcg} / \mathrm{dl}^{(5-23)}$. Se realizan entonces estudios adicionales que evidencian la presencia de hipercortisolismo, por lo cual se realizaron estudios dinámicos de supresión con dosis altas de dexametasona (tabla 1), confirmándose bioquímicamente el diagnóstico de hipercortisolismo endógeno por producción ectópica de ACTH. Se inició manejo con ketoconazol 400 mg cada 12 horas, logrando resolución de la hipocalemia (figura 1).

\section{Caso 2}

Mujer de 68 años de edad con antecedente de enfermedad tromboembólica venosa en el contexto posoperatorio (POP) de resección de un angioma cavernoso en el 2011 que requirió implante de filtro de vena cava inferior (VCI). Historia familiar de cáncer papilar de tiroides en tres hermanos, no había historia de irradiación ni otro antecedente de importancia. Ingresó a la Clínica Las Américas, Medellín, Colombia refiriendo cuadro clínico de dolor en paladar y sensación de masa en cuello. No estaba asociado a ningún proceso infeccioso, no trastornos en el habla, tampoco en la deglución. No refería flushing, no diarrea, no síntomas relacionados con hiperglucemia o hipoglucemia, no lesiones cutáneas. En la revisión por sistemas era llamativa la presencia de edema subjetivo de miembros inferiores, asociado a parestesias y debilidad progresiva de varios meses de evolución. Al examen físico se corroboró lesión palpable en lóbulo tiroideo izquierdo e hipertensión arterial (HTA) de reciente diagnóstico. Durante la hospitalización se

Figura 1. Comportamiento del potasio antes y después de terapia con ketoconazol

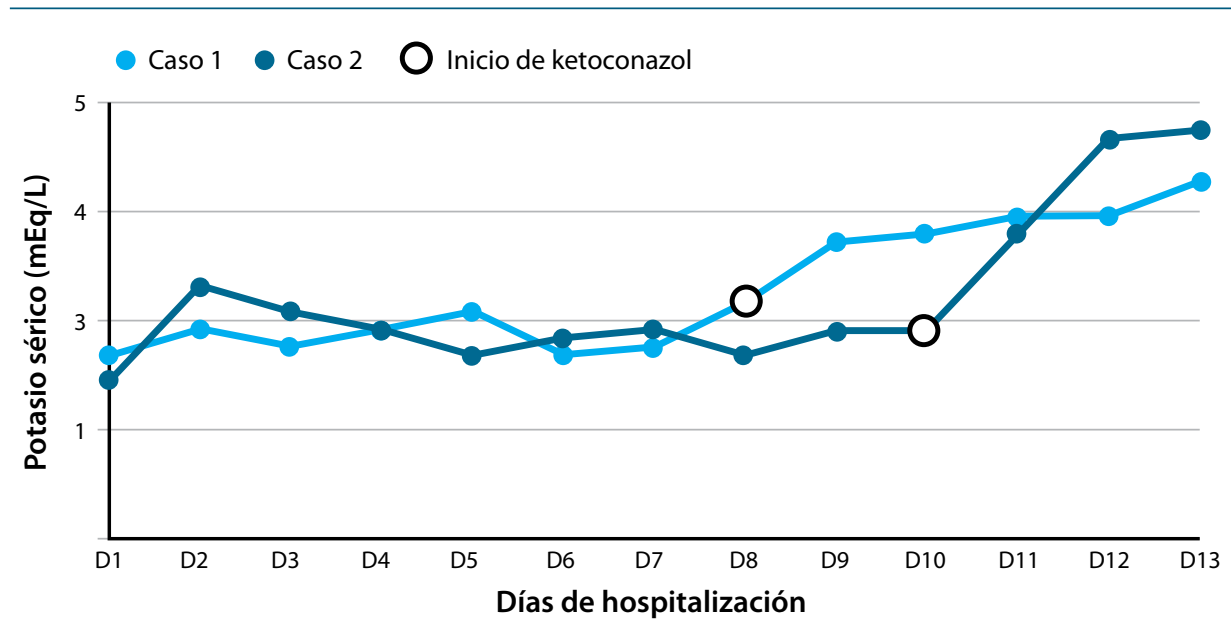

realizó bioquímica sanguínea donde era llamativa la presencia de hipocalemia asociada a alcalosis metabólica (tabla 1). Se inició reposición oral y endovenosa de potasio con evolución clínica tórpida, dada la persistencia de la alteración electrolítica. La ecografía de cuello reportaba pérdida completa de la ecoestructura de la glándula tiroides con disminución de la ecogenicidad y aspecto heterogéneo, múltiples lesiones nodulares algunas confluentes que comprometían ambos lóbulos tiroideos, en el lado derecho la lesión dominante de hasta $22 \mathrm{~mm}$ de diámetro mayor y en el lado izquierdo de $36 \mathrm{~mm}$; estas lesiones descritas como sólidas, vascularizadas, sin microcalcificaciones. Se realiza aspiración con aguja fina (ACAF) de las mismas. En el reporte de patología informan que no se encuentran claramente pseudoinclusiones, tampoco indentaciones nucleares; además algunas células presentan núcleos grandes con pérdida de la relación núcleo citoplasma, planteando la posibilidad de un carcinoma medular de tiroides; pero los niveles séricos de calcitonina y antígeno carcinoembrionario resultaron normales, se indicó manejo quirúrgico de la lesión de tiroides una vez mejorara el estado clínico de la paciente y lograra niveles seguros de potasio en sangre. Como estudios de extensión se realizó tomografía computarizada de tórax (TAC) donde describieron glándula tiroides aumentada de tamaño y de aspecto nodular con adenopatías mediastinales, principalmente de localizacion subcarinal, la dominante de 22 x $28 \mathrm{~mm}$, tráquea y bronquios sin alteraciones, pero en la base pulmonar izquierda se describió un nódulo sólido de bordes espiculados de 12 × 9 mm y tenía además lesiones quísticas simples hepáticas de $3 \mathrm{~mm}$ y $2 \mathrm{~mm}$, acompañadas de dos lesiones sólidas isodensas al parénquima hepático, la dominante en lóbulo hepático derecho de 5 x $5 \mathrm{~cm}$ y la de menor tamaño hacia lóbulo hepático izquierdo de $9 \mathrm{~mm}$. A partir de estos hallazgos en pulmón, se intentó realizar biopsia dirigida por imágenes, pero radiología intervencionista no encontró lesión susceptible de biopsia. Se completó el abordaje diagnóstico con una tomografía por emisión de positrones (PET - CT) con 18 fluordeoxiglucosa (FDG) evidenciándose actividad metabólica anormal localizada entre el cuerpo y la cola del páncreas orientando hacia la posibilidad de un tumor primario a este nivel, acompañado de lesiones hepáticas sugestivas de proceso metastásico (figura 2). Se realizó entonces biopsia de una de las lesiones hepáticas donde se encontraron hallazgos sugestivos de neoplasia neuroendocrina, se realizó inmunohistoquímica (IHQ) para ACTH en esta muestra que resultó ser negativa 
Figura 2. Imágenes de PET - CT con 18 FDG
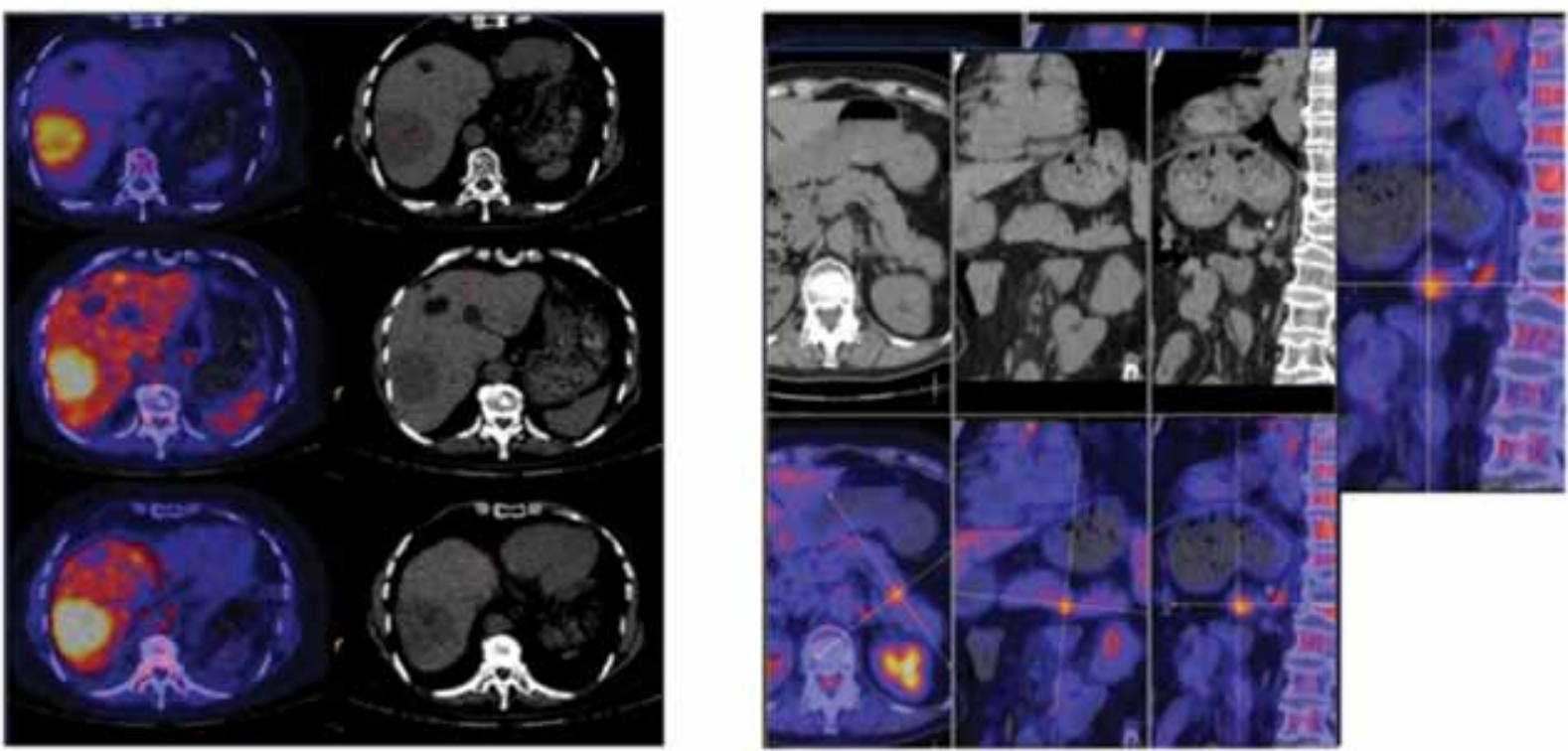

Actividad metabólica predominante en cuerpo y cola de páncreas. En las fases tardías hubo aumento de la actividad, orientando a la posibilidad de lesión primaria. SUV máximo de 2,1. Tiene además múltiples lesiones con actividad metabólica de tipo tumoral a nivel hepático, relacionadas probablemente con proceso metastásico. SUV máximo hasta de 4,1. Por lo menos 4 lesiones nodulares, la de mayor tamaño y actividad localizada hacia el segmento VII de $63 \mathrm{~mm}$. En pulmón izquierdo con SUV máximo de 1,4 pequeño nódulo de $9 \mathrm{~mm}$ localizado en lóbulo inferior. En tórax con SUV máximo de 2,9 a nivel del hilio pulmonar izquierdo, 2 pequeñas adenopatías, la de mayor tamaño de $12 \mathrm{~mm}$. En cuello con SUV máximo de 3,5 se describe lesión localizada en lóbulo tiroideo derecho con diámetro hasta $15 \mathrm{~mm}$.

Figura 3. Biopsia hepática
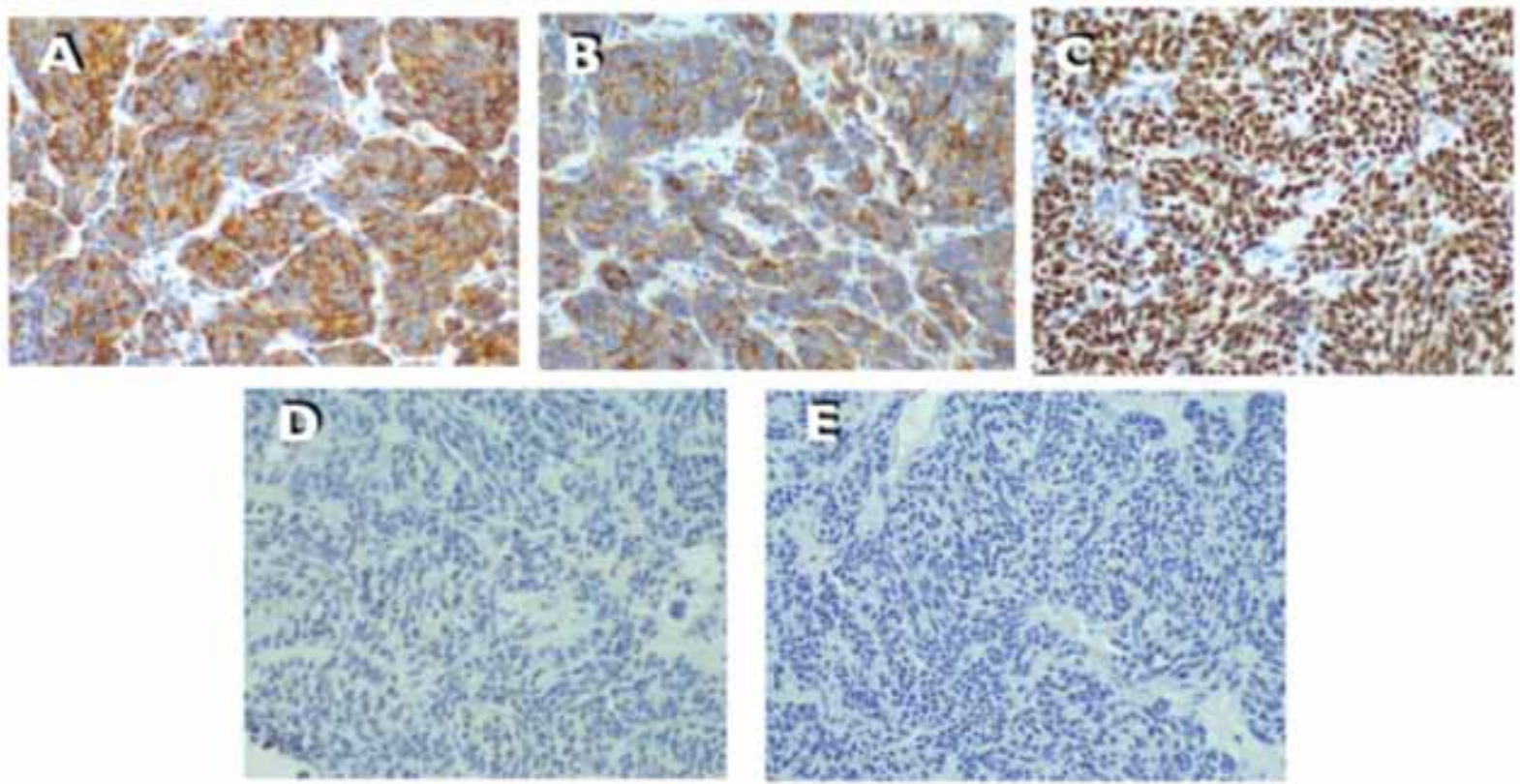

Los cortes muestran fragmentos de tejido infiltrados por una neoplasia constituida por células de tamaño pequeño e intermedio con características neuroendocrinas. Tiene además dos figuras mitóticas por 10 campos de alto poder. Tiñen en forma positiva para marcadores de sinaptofisina (3A), cromogranina (3B) y TTF1 (3C). Ki-67 del 5\%. CDX2 es negativo. Es negativo para calcitonina (3D) y ACTH (3E).

(figura 3). El estudio imaginológico funcional de gamagrafía con octreotide (figura 4) demostró que las lesiones hepáticas conocidas no presentaban captación del radiofármaco, tampoco el nódulo pulmonar del lóbulo inferior izquierdo ni la lesión hipermetabólica descrita en el páncreas, hallazgo que sumado a la presencia de captación de 18FDG en el PET - CT orientaba a la posibilidad de una desdiferenciación o pérdida en la expresión de receptores de somatostatina del tumor neuroen- 

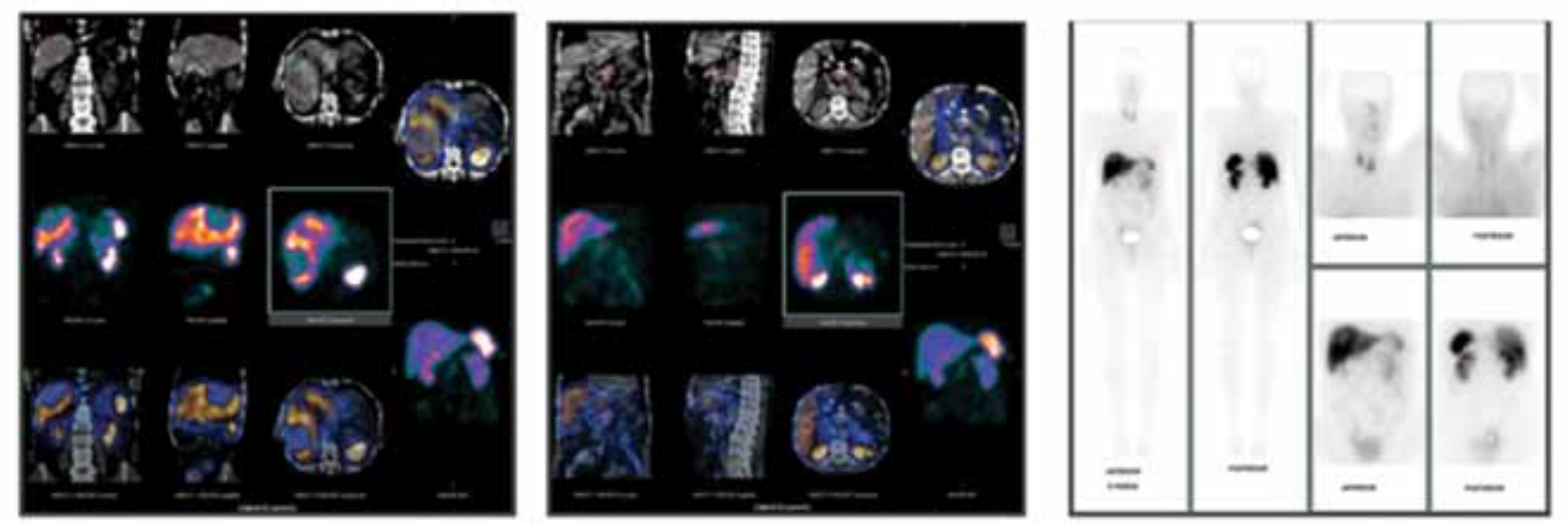

Aumento de la captación en glándula tiroides, donde destaca una lesión nodular hacia el polo inferior del lóbulo izquierdo. Las lesiones hepáticas conocidas, el nódulo pulmonar del lóbulo inferior izquierdo, así como la lesión hipermetabólica descrita en el páncreas no presentan captación del radiofármaco que sugiera expresión de receptores de somatostatina. Bazo accesorio en el flanco izquierdo.

docrino. La RM de abdomen mostraba una imagen hipointensa en T1, hiperintensa en T2 la cual realzaba de manera intensa después del medio de contraste con una zona hipocaptante central por necrosis y restricción en la secuencia difusión con un diámetro mayor de $73 \mathrm{~mm}$ localizada en segmentos VI y VII, otra imagen de $10 \mathrm{~mm}$ de diámetro en segmento II y una en segmento VI de $7 \mathrm{~mm}$ de diámetro de características similares y en el páncreas aunque presentaba aspecto nodular hacia la cola no había una lesión claramente definida. Su evolucion clínica fue tórpida, por la persistencia de hipocalemia a pesar de la suplencia de potasio oral y parenteral, acompañado de dosis altas de espironolactona. Dada la refractariedad al manejo, solicitan valoración por endocrinología, donde se descarta uso de medicamentos asociados a hipocalemia, pérdidas gastrointestinales y tubulopatías asociadas; se planteó entonces la posibilidad de un síndrome de Cushing y se logró confirmar el hipercortisolismo dependiende de ACTH (tabla 1). Como estrategia de control tumoral ante la presencia de un posible tumor neuroendocrino pancreático avanzado, se inició manejo con octreotide LAR $30 \mathrm{mg}$ intramuscular mensual; se decidió entonces realizar pancreatectomía distal, encontrando un tumor en la cola del páncreas de $0,9 \mathrm{~cm}$ unifocal sin evidencia de necrosis con índice mitótico de 5 por 10 campos de alto poder, confinado al tejido pancreático; en la IHQ se corrobora origen neuroendocrino, con marcación negativa para calcitonina y ACTH, sugiriendo de esta manera la posibilidad de secreción ectópica de hormona liberadora de corticotropina (CRH) de la lesión primaria o alguna de las secundarias. Además, teniendo en cuenta que la hipocalemia no mejoraba en presencia de hipercortisolismo documentado dependiente de ACTH de posible origen ectópico, dada la ausencia en RM de cerebro de lesiones a nivel hipofisiario, se decidió realizar prueba terapéutica con ketoconazol $400 \mathrm{mg}$ /día, titulándose hasta 800 mg al día, logrando resolución de la hipocalemia (figura 1).

\section{Discusión}

El conjunto de signos y síntomas explicados por la exposición a niveles altos de glucocorticoides libres en el plasma, endógenos o exógenos, recibe el nombre de síndrome de Cushing (SC) $(1,5)$. El amplio espectro de signos y síntomas que conlleva la enfermedad y las diferentes formas clínicas en que puede presentarse, hacen difícil en ocasiones un abordaje temprano. En términos generales, el SC es una enfermedad rara con una incidencia de 0,2 a 5,0 casos por un millón de habitantes por año y una prevalencia de aproximadamente 39 a 79 casos por millón de habitantes ${ }^{(6)}$.

El SC tradicionalmente se ha clasificado en ${ }^{(7)}$ :

- Pseudocushing: propio de condiciones como el embarazo, alcoholismo, depresión u obesidad.

- Exógeno: por administración de glucocorticoides o sus derivados.

- Endógeno: que a su vez puede ser:

- Dependiente de ACTH (80\%): Secreción hipofisiaria de ACTH (Enfermedad de Cushing), secreción ectópica de ACTH, secreción ectópica de CRH.

- Independiente de ACTH (20\%): Adenoma adrenal, carcinoma adrenal, hiperplasia adrenal nodular pigmentaria.

La secreción ectópica de ACTH cuenta con aproximadamente $4 \%$ a $6 \%$ de los casos de síndrome de Cushing dependiente de ACTH, algunos autores describen, sin embargo, que explica hasta el $20 \%$ de los $\operatorname{casos}^{(8)}$. La realidad es que es un marcador de mal pronóstico oncológico, la causa más común es el carcinoma pulmonar de células pequeñas y el carcinoide bronquial, pero también puede estar relacionada con la secreción hormonal desde tumores neuroendocrinos (TNE) y tumores gastropancreáticos ${ }^{(9)}$ (tabla 2). Esta situación dificulta el abordaje clínico, pues muchos de estos TNE se caracterizan 
Tabla 2. Frecuencia de patologías oncológicas relacionadas con secreción ectópica de ACTH

\begin{tabular}{l|c}
\hline \multicolumn{1}{c|}{ Condición clínica } & $\begin{array}{c}\text { Frecuencia de } \\
\text { ACTH ectópico }\end{array}$ \\
\hline Carcinoma pulmonar de células pequeñas & $3,3 \%-50 \%$ \\
\hline Carcinoide bronquial & $3,3 \%-54,8 \%$ \\
\hline Carcinoide tímico & $5 \%-42 \%$ \\
\hline Tumores neuroendocrinos pancreáticos & $7,5 \%-25 \%$ \\
\hline Cáncer medular de tiroides & $2 \%-8 \%$ \\
\hline Feocromocitoma & $2,5 \%-25 \%$ \\
\hline Tumores con primario desconocido & $12 \%-36 \%$ \\
\hline
\end{tabular}

por la producción de una o varias hormonas diferentes a la ACTH o en menor proporción $\mathrm{CRH}$, causando diferentes síndromes clínicos que se sobreponen al del hipercortisolismo ${ }^{(10)}$; existen reportes de secreción concomitante de ambas hormonas ${ }^{(11)}$. Se ha descrito que puede haber tumores desconocidos primarios productores de ACTH hasta en un $12 \%-36 \%{ }^{(12)}$ e inclusive producción ectópica de ACTH en tumores sin diferenciación neuroendocrina hasta en el 8\% de los casos (adenocarcinoma de ovario, cérvix, anorrectal, pulmón, próstata y neuroblastoma) ${ }^{(3,12)}$. En general, estos tumores productores de ACTH secretan de manera desproporcionada precursores de la propiomelanocortina (POMC) y se ha considerado la relación POMC/ACTH como un marcador de agresividad tumoral ${ }^{(13)}$.

En los casos de SC por secreción ectópica de ACTH, el compromiso suele ser usualmente igual en hombres y mujeres, con una relación 1:1 o 2:1, a diferencia de lo que ocurre en el SC de otras etiología, donde predomina el compromiso en mujeres. La edad de presentación suele ser alrededor de la cuarta década de la vida ${ }^{(3,12)}$, un poco más tardía en comparación con otros casos de SC. Dentro de los hallazgos clínicos en pacientes con síndrome de Cushing por secreción ectópica de ACTH, se describe la presencia de infecciones oportunistas reflejo del estado de inmunosupresión crónica hasta en el $29 \%$, HTA hasta en un $64 \%$ de los pacientes, ganancia de peso en un $82 \%$, debilidad muscular 94\%, diabetes mellitus en un 53\% y alteraciones electrolíticas donde la hipocalemia puede estar presente hasta en $64 \%$ de los $\operatorname{casos}^{(9)}$; otras series describen una frecuencia que puede ir desde el $42 \%$ hasta el $70 \%{ }^{(3,12)}$. Inclusive, desde el punto de vista clínico es importante tener en cuenta que muchas características típicas del hipercortisolismo, pueden confundirse con las del propio cáncer, como lo es el desgaste muscular, la marcada astenia y la perdida objetiva de peso ${ }^{(14)}$. Sin embargo, la velocidad de instauración y agresividad de signos y síntomas en el síndrome de Cushing por secreción ectópica de ACTH dependen del tipo de tumor y tejido productor de la hormona, usualmente hay una instauración más insidiosa del cuadro en casos asociados con tumores neuroendocrinos de otros órganos diferentes al pulmón o bronquiales (células pequeñas del pulmón) don- de predominan síntomas asociados a trastornos psiquiátricos, osteoporosis, infecciones y aumento en la incidencia de enfermedad tromboembólica, tal y como había sucedido en el caso $2^{(1-3)}$. Sin embargo, es importante tener en cuenta que más que el tiempo de instauración, el mayor determinante de las características clínicas es el grado de hipercortisolemia ${ }^{(9)}$. En los casos de instauración rápida del hipercortisolismo, el paciente no suele tener el fenotipo típico del SC.

Ante la presencia de hipocalemia como manifestación principal, las causas usuales pueden ser determinadas con los datos de la historia clínica (uso de diuréticos, vómito o diarrea); sin embargo, en algunos casos, la causa no es tan evidente y habría que descartar entre causas menos comunes la acidosis tubular renal, cetoacidosis diabética, hiperinsulinemia, hiperaldosteronismo primario y producción ectópica de ACTH. En el caso del síndrome de Cushing, el exceso de cortisol, además de su efecto glucocorticoide, ejerce un efecto indirecto mineralocorticoide a través del sistema renina angiotensina aldosterona (SRAA) explicando de esta manera no solo la HTA sino también la hipocalemia; esta última activa en las células intercaladas A adyacentes a las células principales renales, la bomba $\mathrm{H}^{+} \mathrm{K}^{+}$ATPasa, secretándose hidrogeniones en la orina a cambio de iones de potasio. Este fenómeno explicaría la asociación típica con alcalosis metabólica ${ }^{(15)}$ tal y como se describió en los casos clínicos presentados.

En el abordaje diagnóstico del síndrome de Cushing, lo primero que debe hacerse es excluir el uso exógeno de glucocorticoides a través de una historia clínica completa ${ }^{(7)}$. Posteriormente se realizarán diferentes pruebas de tamización para confirmar la producción endógena de cortisol, seguidas de aquellas que permiten establecer cuál es su origen y finalmente ofrecer un tratamiento apropiado. Dentro de las pruebas de tamización inicial (cortisol libre en orina de 24 horas en al menos dos mediciones diferentes, cortisol libre salival a las 23 h, supresión con $1 \mathrm{mg}$ de dexametasona, supresión con $2 \mathrm{mg}$ de dexametasona en $48 \mathrm{~h}$ ) hay que tener en cuenta que ninguna de ellas tiene una especificidad del $100 \%$ y la recomendación universal es realizar una prueba inicial altamente sensible y luego excluir con otra altamente específica ${ }^{(7)}$, así dos de cuatro pruebas positivas confirmarán el hipercortisolismo en pacientes con alta probabilidad pretest. Se debe anotar que en el SC ectópico, el valor del cortisol libre en orina suele ser mucho mayor que en los casos de SC causado por adenomas hipofisiarios $^{(1,9,16,17)}$.

El siguiente paso es determinar los niveles de ACTH plasmática $^{(7)}(\mathrm{VN}: 20$ a $80 \mathrm{pg} / \mathrm{ml}$ a las 08:00 h). Valores por debajo de $5 \mathrm{pg} / \mathrm{ml}$ indican una causa de origen adrenal, mientras que valores por encima de $20 \mathrm{pg} / \mathrm{ml}$ sugieren dependencia del cortisol a la secreción de ACTH, de los cuales el 80\% corresponden a una enfermedad de Cushing, pero cuando estos están por encima de $200 \mathrm{pg} / \mathrm{ml}$ hay que pensar en secreción ectópica de ACTH; sin embargo, su ausencia no lo descarta ${ }^{(8)}$; por lo cual es 
importante tener en cuenta que no hay un punto de corte que ayude a distinguir enfermedad de Cushing de producción ectópica de ACTH e inclusive no hay discrepancia en los niveles de ACTH entre tumores ocultos vs. tumores manifiestos, indicando que no hay una relación directa entre el tamaño tumoral y la producción de $\mathrm{ACTH}^{(3)}$. Si bien una resonancia magnética (RM) de silla turca sería el examen ideal ante la presencia de un síndrome de Cushing ACTH dependiente, hay que tener en cuenta que solamente los tumores hipofisiarios mayores de $6 \mathrm{~mm}$ podrán ser considerados causantes de la enfermedad de Cushing y que hasta el $10 \%$ de la población normal puede tener incidentalomas hipofisiarios que no están relacionados con el proceso patológico ${ }^{(7,9,12)}$.

Por esta razón se recomienda realizar algunas pruebas dinámicas complementarias para evitar cirugías transesfenoidales innecesarias; dentro de estas pruebas se incluye la supresión con dosis altas de dexametasona (8 $\mathrm{mg}$ a las 23 horas) ${ }^{(7)}$ para diferenciar los casos de ACTH dependiente, partiendo del hecho de que los tumores hipofisiarios son menos autónomos que los tumores ectópicos, pues hasta un $90 \%$ de estos últimos no se dejan suprimir aunque $10 \%$ a $15 \%$ sí lo harán ${ }^{(3,9,17)}$ y a tener en cuenta que los tumores carcinoides (usualmente los bronquiales) pueden también responder a la retroalimentación negativa ${ }^{(3)}$. Con esta prueba se espera que tras la administración de dexametasona a dosis altas la noche anterior $(8 \mathrm{mg}$ a las 11 p.m.), los niveles de cortisol sérico a las 8 a.m. estén $<5$ $\mathrm{mcg} / \mathrm{dl}$ o en su defecto que haya habido una reducción de más del 50\% de los niveles séricos respecto al valor basal, lo cual indicaría la posibilidad de enfermedad de Cushing en contraposición a un tumor ectópico, situación que no sucedió en ninguno de los dos casos descritos. Si hay supresión de cortisol sérico tras la prueba y se sigue pensando en la posibilidad de secreción ectópica de $\mathrm{ACTH}$, podría realizarse otro test dinámico que consiste en la administración endovenosa de CRH. Desde el punto de vista biológico, los tumores ectópicos productores de ACTH no tienen receptores de CRH, así, tras la administración de CRH ovina ( $1 \mathrm{mcg} / \mathrm{kg}$ ) o humana (100 mcg) intravenosa dosis única a las 08:00 h con posterior medición secuencial de cortisol sérico y ACTH desde los -15 minutos hasta los 60 a 120 minutos posadministración, se espera que haya un aumento > del 35\% en los niveles de ACTH y del 14\% al 20\% en los niveles de cortisol sérico a los 15 a 30 minutos en el caso de que se trate de una enfermedad de Cushing, con una sensibilidad reportada del $93 \%$ y una especificidad del $100 \%{ }^{(18)}$. En los casos de SC ectópico no habrá respuesta o el aumento será mínimo, usualmente menor de 1,5 veces ${ }^{(9)}$. Existen otros test de estimulación de menor disponibilidad en nuestro medio, entre los que se incluyen test de estimulación con desmopresina, hexarelina, secretagogos de la GH, combinación de CRH y desmopresina o pruebas dinámicas con metirapona ${ }^{(7)}$.

A pesar de la existencia de estas pruebas dinámicas que ayudan a orientar frente a una enfermedad de Cushing vs. un síndrome de Cushing ACTH dependiente ectópico, el examen con mayor capacidad discriminatoria, de casi el $100 \%$ es el cateterismo de senos venosos petrosos, el cual permite determinar si la secreción de ACTH es central o periférica e intenta lateralizar la lesión. Este examen consiste en la medición de la ACTH que drena directamente de la hipófisis y con base en esto se determina el gradiente de ACTH a nivel central vs. periférico. Puede hacerse con medición basal de ACTH o tras estímulo con CRH. Un gradiente de ACTH seno petroso/ vena periférica menor 2 veces sin estímulo de CRH y menor de 3 veces con estímulo de CRH, obliga a buscar una secreción ectópica de ACTH, con una especificidad del 95\% al 100\%(19). Como alternativa a la $\mathrm{CRH}$, difícil de conseguir en nuestro medio, se puede usar la desmopresina ${ }^{(20)}$ (sensibilidad del 92,1\% y especificidad del $100 \%$ ) con una interpretación igual a como se mencionó con $\mathrm{CRH}^{(21)}$. Sin embargo, al ser un examen invasivo y requerir personal experto, no se realiza de forma rutinaria $^{(22)}$, pero en casos seleccionados debe realizarse en centros especializados.

Una vez se realiza la confirmación bioquímica de un tumor productor de ACTH ectópico, se debe realizar un estudio imaginológico para confirmar su ubicación anatómica; no se ha definido cuál es la mejor modalidad de estudio para detectar estos tumores. Se puede usar la tomografía axial computarizada (TAC) toracoabdominal con una sensibilidad del 53\% o una RM con una sensibilidad del $37 \%$. Se recomienda iniciar con estudios a nivel torácico, dado que la mayoría de los tumores productores de ACTH se encuentran en este lugar. Los estudios de imágenes a nivel abdominal muy raramente detectan un tumor oculto, y es más factible la determinación de metástasis a nivel hepático. De ser estos negativos (lo que puede ocurrir hasta en el $20 \%$ de los casos), podría considerarse el uso de estudios imaginológicos funcionales de medicina nuclear (análogos de receptores de somatostatina) como alternativa diagnóstica. Se debe tener en cuenta que el hipercortisolismo crónico puede generar una regulación a la baja de los receptores de somatostatina, pudiéndose reportar falsos negativos con el Octreoscan; fenómeno que mejorará una vez se haga tratamiento médico inicial del hipercortisolismo, además no todos los tumores tienen receptores o los subtipos para los cuales los análogos de somatostatina son más afines (receptores 2 y 5), y pueden existir falsos positivos en patologías no neoplásicas. Es probable que este fenómeno de "regulación a la baja" sea lo que haya sucedido en el caso 2 y, por esto, este estudio funcional no hubiera sido concluyente. Las diferentes series describen que la sensibilidad de la prueba va del $50 \%$ al $60 \%{ }^{(23,24)}$. Como alternativa se ha venido usando el PET - CT, donde el marcador galio (68 Ga - DOTATATE) ha demostrado que puede ser útil en la localización de tumores no detectados en otras imágenes, inclusive con una sensibilidad mayor cuando se compara con el marcador tradicional 18 FDG $(82 \%$ vs. $66 \%$ ) en tumores neuroendocrinos bien diferenciados ${ }^{(25)}$. 
También se ha usado el marcador 18F - DOPA con buenos resultados para otro tipo de neoplasias ${ }^{(26)}$.

En los casos presentados, si bien el antecedente de carcinoma de células pequeñas de pulmón aumentaba la sospecha de un síndrome paraneoplásico en el caso 1, en el caso 2 el abordaje diagnóstico requirió el uso estructurado de pruebas bioquímicas, hormonales e imaginológicas para confirmar el diagnóstico de un TNE grado 2 . En ambos, el síntoma cardinal fue la presencia de hipocalemia asociada a alcalosis metabólica y tendencia hipertensiva. Se presenta en este sentido un flujograma diagnóstico que parte del hallazgo de hipocalemia (figura 5).

El tratamiento dependerá de la causa encontrada, siendo el manejo quirúrgico de la lesión primaria la primera opción terapéutica, con lo cual se alcanzan tasas de curación variables $(12 \% \text { a } 71 \%)^{(12)}$. En ninguno de los dos casos aquí descritos pudo ofrecerse esta opción terapéutica, dado lo avanzado de la enfermedad. Usualmente el pronóstico depende de la neoplasia de base y de la presencia de metástasis al momento del diagnóstico. Dentro de las opciones farmacológicas se incluye el uso de antagonistas del receptor de glucocorticoides (mifepristona), adrenolíticos (mitotane), inhibidores de la síntesis de glucocorticoides (ketoconazol, metirapona) o en casos muy avanzados sin respuesta clínica se puede realizar adrenalectomía bilateral, idealmente por abordaje laparoscópico. Otras opciones terapéuticas están basadas en que estos tumores productores de ACTH expresan también receptores de somatostatina y de dopamina, y al actuar sobre ellos se regula la producción de cortisol; en este sentido se han reportado casos manejados con octreotide o cabergolina. Usualmente estas terapias no tienen control sobre el crecimiento tumoral. No cabe duda de que parte del manejo incluye un abordaje multidisciplinario, contado el manejo oncoespecífico de la neoplasia de base ${ }^{(5,7,8,12)}$.

Figura 5. Flujograma diagnóstico de pacientes con hipocalemia y sospecha de hipercortisolismo por secreción ectópica de ACTH

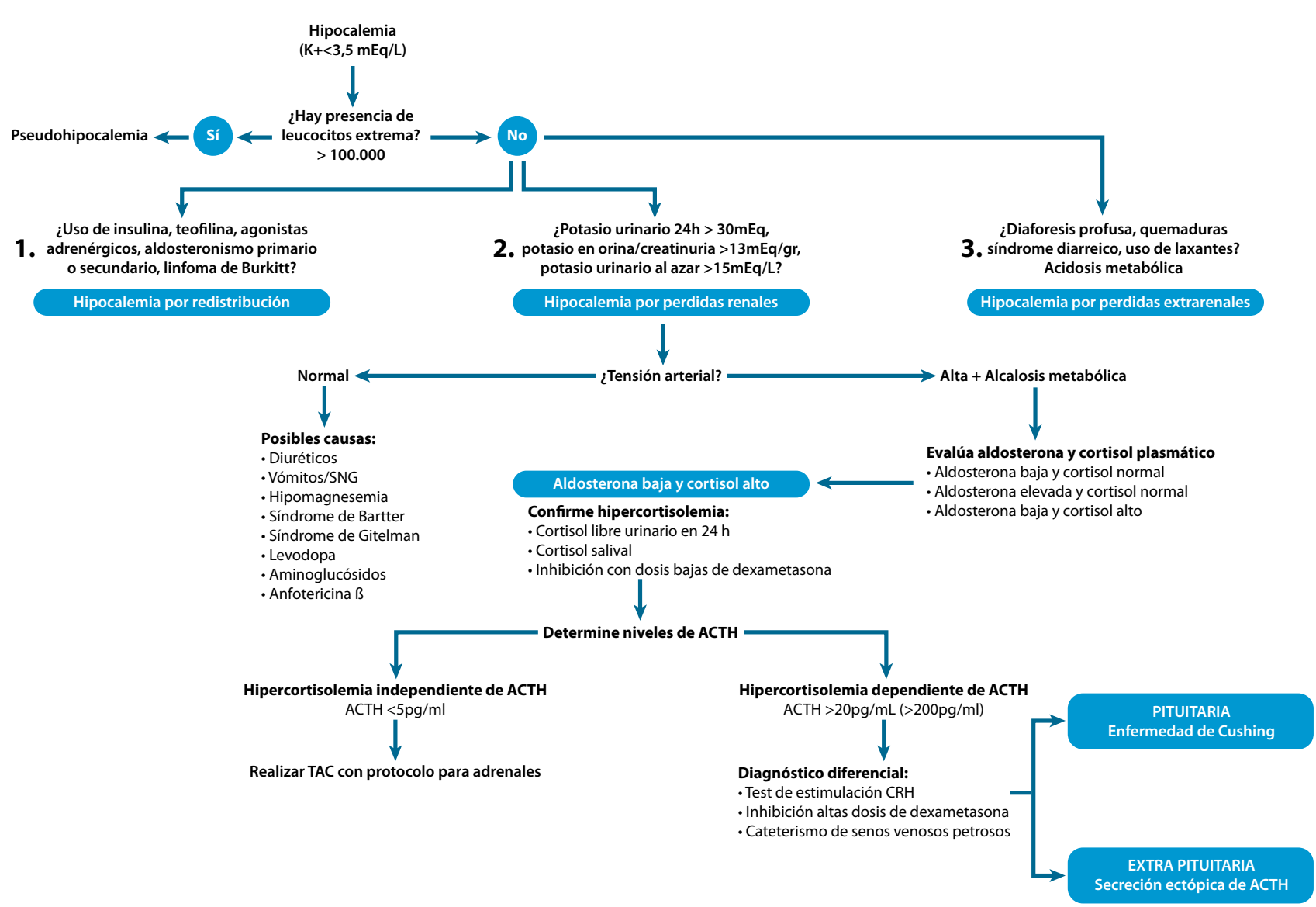




\section{Conclusión}

La hipocalemia refractaria al manejo convencional con reposición de potasio oral e intravenoso y el uso de medicamentos ahorradores de potasio en pacientes oncológicos debe llevarnos a sospechar la presencia de un síndrome de Cushing ectópico; estos pacientes tienden a tener mayores alteraciones hidroelectrolíticas y no desarrollan un fenotipo clásico como en otros escenarios de hipercortisolismo. Es importante conocer cómo debe realizarse el abordaje diagnóstico en estos casos y las diferentes pruebas de laboratorio e imaginológicas que se deben solicitar de tamización y confirmación. El tratamiento quirúrgico del tumor causante de la secreción de la ACTH es la mejor opción, pero deberá considerarse el manejo farmacológico en aquellos pacientes en los cuales esto no es posible; a pesar de las limitaciones por la poca disponibilidad de los medicamentos indicados en estos casos en nuestro medio, disponemos de ketoconazol como bloqueador de la esteroidogénesis, el cual ha mostrado ser útil en casos de hipocalemia refractaria causada por síndrome de Cushing ectópico dependiente de ACTH. No debemos olvidar que el pronóstico oncológico va a estar determinado directamente por la neoplasia de base y el manejo deberá ser multidisciplinario (endocrinología, oncología, medicina interna, cirugía endocrina, patología, biología molecular, entre otros) para permitir un abordaje integral y así lograr la instauración de un tratamiento oportuno y adecuado que impacte en la morbilidad y mortalidad del paciente.

\section{Fuentes de financiación}

No se requirió ninguna fuente de financiación

\section{Conflicto de interés}

Los autores no declaran ningún conflicto de interés

\section{Referencias}

1. Pivonello R, De Martino MC, De Leo M, Lombardi G, Colao A. Cushing's Syndrome. Endocrinol Metab Clin North Am. Marzo de 2008;37(1):135-49.

2. Kreitschmann-Andermahr I, Psaras T, Tsiogka M, Starz D, Kleist B, Siegel S, et al. From first symptoms to final diagnosis of Cushing's disease: experiences of 176 patients. Eur J Endocrinol. Febrero 6 de 2015;172(3):285-9.

3. Ilias I, Torpy DJ, Pacak K, Mullen N, Wesley RA, Nieman LK. Cushing's Syndrome Due to Ectopic Corticotropin Secretion: Twenty Years' Experience at the National Institutes of Health. J Clin Endocrinol Metab. Agosto de 2005;90(8):4955-62.

4. Findling JW, Raff H. DIAGNOSIS OF ENDOCRINE DISEASE: Differentiation of pathologic/neoplastic hypercortisolism (Cushing's syndrome) from physiologic/non-neoplastic hypercortisolism (formerly known as pseudo-Cushing's syndrome). Eur J Endocrinol. Mayo de 2017;176(5):R205-16.

5. Prague JK, May S, Whitelaw BC. Cushing's syndrome. BMJ. Marzo 27 de 2013;346(mar27 3):f945-f945.

6. Lacroix A, Feelders RA, Stratakis CA, Nieman LK. Cushing's syndrome. The Lancet. Agosto de 2015;386(9996):913-27.

7. Nieman LK, Biller BMK, Findling JW, Newell-Price J, Savage MO, Stewart PM, et al. The Diagnosis of Cushing's Syndrome: An Endocrine Society Clinical Practice Guideline. J Clin Endocrinol Metab. Mayo de 2008;93(5):1526-40.

8. Gutiérrez-Restrepo J, Román-González A. Síndrome de Cushing ectópico: revisión de la literatura. Rev Colomb Cancerol. Octubre de 2016;20(4):175-82.

9. Isidori AM, Kaltsas GA, Pozza C, Frajese V, Newell-Price J, Reznek RH, et al. The Ectopic Adrenocorticotropin Syndrome: Clinical Features, Diagnosis, Management, and Long-Term Follow-Up. J Clin Endocrinol Metab. Febrero de 2006;91(2):371-7.

10. Oronsky B, Ma PC, Morgensztern D, Carter CA. Nothing But NET: A Review of Neuroendocrine Tumors and Carcinomas. Neoplasia. Diciembre de 2017;19(12):991-1002.

11. Asa SL, Kovacs K, Vale W, Petrusz P, Vecsei P. Immunohistologic localization of corticotrophin-releasing hormone in human tumors. Am J Clin Pathol. Marzo de 1987;87(3):327-33.

12. Alexandraki KI, Grossman AB. The ectopic ACTH syndrome. Rev Endocr Metab Disord. Junio de 2010;11(2):117-26.

13. Raffin-Sanson ML, Massias JF, Dumont C, Raux-Demay MC, Proeschel MF, Luton JP, et al. High plasma proopiomelanocortin in aggressive adrenocorticotropin-secreting tumors. J Clin Endocrinol Metab. Diciembre de 1996;81(12):4272-7.

14. Aschner P, Jácome A, Jiménez L, de Anzola MI. Síndrome de secreción ectópica de ACTH por un carcinoma de células en avena del pulmón. Acta Medica Colombiana. Diciembre de 1985;10(6):259.

15. Lee Hamm L, Hering-Smith KS, Nakhoul NL. Acid-Base and Potassium Ho- meostasis. Semin Nephrol. Mayo de 2013;33(3):257-64.

16. Barbosa SL-S, Rodien P, Leboulleux S, Niccoli-Sire P, Kraimps J-L, Caron P, et al. Ectopic adrenocorticotropic hormone-syndrome in medullary carcinoma of the thyroid: a retrospective analysis and review of the literature. Thyroid Off J Am Thyroid Assoc. Junio de 2005;15(6):618-23.

17. Kakade H, Kasaliwal R, Jagtap V, Bukan A, Budyal S, Khare S, et al. Ectopic ACTH-Secreting Syndrome: A Single-Center Experience. Endocr Pract. Noviembre de 2013;19(6):1007-14.

18. Newell-Price J, Morris DG, Drake WM, Korbonits M, Monson JP, Besser GM, et al. Optimal Response Criteria for the Human CRH Test in the Differential Diagnosis of ACTH-Dependent Cushing's Syndrome. J Clin Endocrinol Metab. Abril de 2002;87(4):1640-5.

19. Colao A, Faggiano A, Pivonello R, Pecori Giraldi F, Cavagnini F, Lombardi G, et al. Inferior petrosal sinus sampling in the differential diagnosis of Cushing's syndrome: results of an Italian multicenter study. Eur J Endocrinol. Mayo de 2001;144(5):499-507.

20. Castinetti F, Morange I, Dufour H, Jaquet P, Conte-Devolx B, Girard N, et al. Desmopressin test during petrosal sinus sampling: a valuable tool to discriminate pituitary or ectopic ACTH-dependent Cushing's syndrome. Eur J Endocrinol. Septiembre 1 de 2007;157(3):271-7.

21. Colao A, Faggiano A, Pivonello R, Pecori Giraldi F, Cavagnini F, Lombardi G, et al. Inferior petrosal sinus sampling in the differential diagnosis of Cushing's syndrome: results of an Italian multicenter study. Eur J Endocrinol. Mayo de 2001;144(5):499-507.

22. Loriaux DL. Diagnosis and Differential Diagnosis of Cushing's Syndrome. Longo DL, editor. N Engl J Med. Abril 13 de 2017;376(15):1451-9.

23. Tabarin A, Valli N, Chanson P, Bachelot Y, Rohmer V, Bex-Bachellerie V, et al. Usefulness of Somatostatin Receptor Scintigraphy in Patients with Occult Ectopic Adrenocorticotropin Syndrome. J Clin Endocrinol Metab. Abril de 1999;84(4):1193-202.

24. Tsagarakis S, Christoforaki M, Giannopoulou H, Rondogianni F, Housianakou I, Malagari C, et al. A Reappraisal of the Utility of Somatostatin Receptor Scintigraphy in Patients with Ectopic Adrenocorticotropin Cushing's Syndrome. J Clin Endocrinol Metab. Octubre de 2003;88(10):4754-8.

25. Kayani I, Bomanji JB, Groves A, Conway G, Gacinovic S, Win T, et al. Functional imaging of neuroendocrine tumors with combined PET/CT using68GaDOTATATE (DOTA-DPhe1,Tyr3-octreotate) and18F-FDG. Cancer. Junio 1 de 2008;112(11):2447-55.

26. Kauhanen S, Seppanen M, Ovaska J, Minn H, Bergman J, Korsoff P, et al. The clinical value of [18F]fluoro-dihydroxyphenylalanine positron emission tomography in primary diagnosis, staging, and restaging of neuroendocrine tumors. Endocr Relat Cancer. Noviembre 11 de 2008;16(1):255-65. 